\title{
SPANISH TERMS
}

\begin{tabular}{|c|c|c|c|}
\hline aguada & pond & glorieta $(s)$ & traffic circle(s) \\
\hline autopista & $\begin{array}{l}\text { motorway (usually } \\
\text { toll) }\end{array}$ & $\begin{array}{l}\text { grutas } \\
\text { lancha }(s)\end{array}$ & $\begin{array}{l}\text { caves } \\
\text { open fishing }\end{array}$ \\
\hline barranca & large canyon & & launch(es) \\
\hline bienvenido & welcome & libre & free \\
\hline cabañas & cabins & malecon & waterfront street \\
\hline cambios & exchange booths & & or boulevard \\
\hline cascadas & falls & mercado & market \\
\hline caseta & small building, booth & microondas & radio towers \\
\hline cenote & limestone sinkhole & $\operatorname{milpa}(s)$ & agricultural plot(s) \\
\hline centro & center/downtown & mirador & overlook \\
\hline chivizcoyo & $\begin{array}{l}\text { Bearded Wood- } \\
\text { Partridge }\end{array}$ & $\begin{array}{l}\operatorname{norte}(s) \\
\operatorname{palapa}(s)\end{array}$ & $\begin{array}{l}\text { norther(s) } \\
\text { thatched open hut(s) }\end{array}$ \\
\hline colectivo & $\begin{array}{l}\text { collective (communal) } \\
\text { taxi }\end{array}$ & $\begin{array}{l}\text { Pemex } \\
\text { periferico }\end{array}$ & $\begin{array}{l}\text { gas station } \\
\text { bypass }\end{array}$ \\
\hline colectivo combis & VW minibuses & propina & tip \\
\hline cumbre & summit & pueblo & village, town \\
\hline cuota & toll & puente & bridge \\
\hline Don & $\begin{array}{l}\text { term of respect for } \\
\text { (usually oder) men }\end{array}$ & $\begin{array}{l}\text { puerto } \\
\text { rancho(s) }\end{array}$ & $\begin{array}{l}\text { port } \\
\text { ranch(es) }\end{array}$ \\
\hline Doña & $\begin{array}{l}\text { term of respect for } \\
\text { (usually older) women }\end{array}$ & río & river \\
\hline ejido & $\begin{array}{l}\text { common land/ } \\
\text { cooperative village }\end{array}$ & zócalo & town square, plaza \\
\hline este & estuary & Zona Hotelera & hotel zone \\
\hline fince & plantation & & \\
\hline
\end{tabular}

